\title{
Numerical study of the phase behavior of rodlike colloids with attractive interactions
}

\author{
Peter G. Bolhuis \\ FOM Institute for Atomic and Molecular Physics, Kruislaan 407, 1098 SJ Amsterdam, The Netherlands \\ Alain Stroobants \\ Van't Hoff Laboratory, Padualaan 8, 3584 CH, Utrecht, The Netherlands \\ Daan Frenkel \\ FOM Institute for Atomic and Molecular Physics, Kruislaan 407, 1098 SJ Amsterdam, The Netherlands
}

Henk N. W. Lekkerkerker

Van't Hoff Laboratory, Padualaan 8, 3584 CH, Utrecht, The Netherlands

(Received 14 January 1997; accepted 21 April 1997)

\begin{abstract}
We examine the influence of attractive interactions on the phase behavior of rodlike colloids. We model the rodlike particles by spherocylinders, for which the phase diagram, in the absence of attraction, is known for arbitrary aspect ratio. We consider the case that the attraction is due to depletion forces caused by the addition of nonadsorbing polymer. The range of this attraction is determined by the size of the polymer. If the radius of gyration of the polymer is small compared to the diameter of the rods, we can model the polymer-induced attraction by a suitable generalization of the square-well model for spherical particles. However, for longer ranged attractions, pairwise additive attractions lead to phase behavior that is very different from what is found when the nonadditivity of depletion forces is taken into account. In our simulations, we find evidence for demixing transitions in the isotropic, nematic and solid phases. We compare our simulation results with predictions based on the perturbation theory of Lekkerkerker and Stroobants [Nuovo Cimento D 16, 949 (1994)]. A crucial input in this theory is the so-called free-volume fraction of the hard spherocylinder reference system. In the work of Lekkerkerker and Stroobants, this quantity is estimated using scaled-particle theory. We test the validity of this approach by comparing it to numerical results for the free-volume fraction. (c) 1997 American Institute of Physics. [S0021-9606(97)50129-2]
\end{abstract}

\section{INTRODUCTION}

The isolation and purification of rodlike viruses, such as the tobacco-mosaic virus (TMV), can be facilitated considerably by addition of nonadsorbing polymers. ${ }^{1}$ This fact has been known to experimentalists for over half a century. However, as these experiments focused on the purification of the virus, little attention was paid to the physics underlying the phase behavior of such mixtures of polymers and rodlike colloids. Yet, TMV dispersions are, in a sense, model colloids - the virus particles behave to a first approximation as hard rods. And, in fact, TMV dispersions exhibit the kind of liquid-crystalline ordering expected for hard rods. ${ }^{2} \mathrm{Nev}-$ ertheless, a direct comparison between experiments on TMV and theories for hard rod suspensions remains limited because the length of the virus particles cannot be varied at will. Therefore, in recent years, much effort has been invested in the development of reliable techniques to synthesize sterically stabilized rodlike colloidal boehmite needles with a variable length-to-width ratio. ${ }^{3}$ Dispersions of such particles could serve as a model system for rodlike colloids, much in the same way as dispersions of spherical PMMA and silica particles act as prototypical experimental realizations of hard-sphere fluids. The boehmite rods have the added advantage that the length-to-width ratio can be varied. Thus far, these boehmite suspensions are still fairly polydis- perse, but the situation is likely to improve as more sophisticated colloid synthesis techniques are developed. Buitenhuis et al. ${ }^{4}$ conducted experiments on a mixture of polystyrene and boehmite rods with an aspect ratio of 6.4 , sterically stabilized with polyisobutene ${ }^{5}$ and dispersed in an organic solvent. For a polymer radius of gyration which was about $80 \%$ of the rod diameter they observed phase separation into two isotropic phases at sufficiently high polymer concentration.

In the present paper, we consider the effect of attractive interactions on the phase behavior of rodlike colloids. As most of the controlled experimental studies to date have been performed on systems where the attraction is due to polymerinduced depletion forces, we pay mainly attention to this specific interaction. For short-ranged interactions (i.e., small polymers), depletion forces are, to a good approximation pairwise additive. Hence, in that limit we can describe the depletion interaction using an appropriately chosen pair potential. However, for longer-ranged interactions, the behavior of systems of rods with a pairwise additive attraction turns out to be very different from what is observed when the nonadditive nature of depletion forces is taken into account. We therefore consider the two cases separately.

In what follows, we discuss the theory and simulation of polymer-colloid mixtures in the context of a hard particle model. To be precise, we model the colloids as hard sphero- 


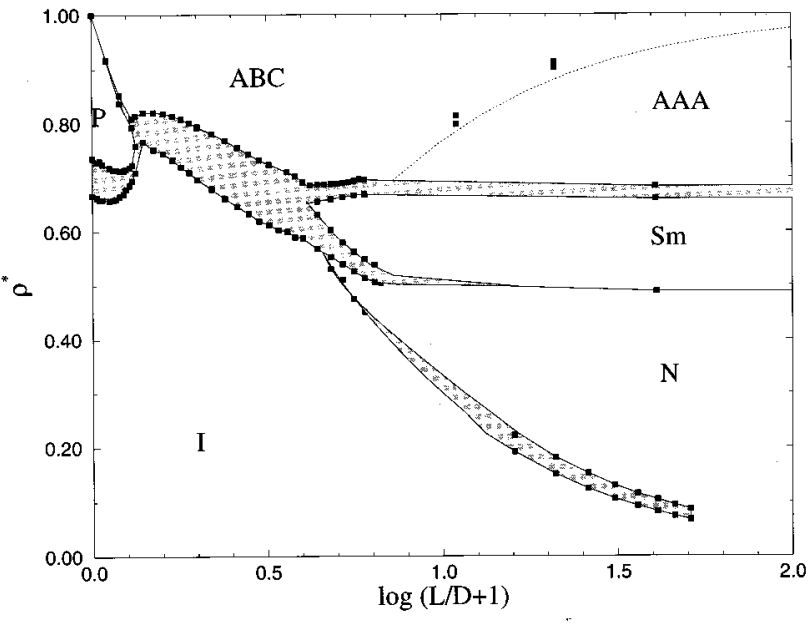

FIG. 1. Phase diagram of hard spherocylinders with $L / D$ between 0 and 60 . In order to give equal emphasis to all parts of the phase diagram, we have plotted $\rho^{*}$ as a function of $\log (L / D+1)$. All two-phase regions are shown shaded. In the figure, the following phases can be distinguished: the lowdensity isotropic liquid I, the high-density orientationally-ordered solid $A B C$, the low- $L / D$ plastic solid $P$ and, for $L / D>3.7$, the nematic $\mathrm{N}$ and smectic-A phases SmA. For $L / D>7$ an $A A A$ stacked solid becomes stable between the smectic and the $A B C$ stacked solid phase. The dashed line is a crude estimate for the first order $A A A-A B C$ transition. For more details see Ref. 9. In the present paper we focus on the case $L / D=5$.

cylinders of length $L$ and diameter $D$. The shape of these particles is characterized by the nonsphericity $L / D$ (zero for spheres) or, equivalently, the aspect ratio $L / D+1$. We stress that this model, although simple, is quite realistic for many lyotropic liquid crystalline materials. ${ }^{2}$ The present paper focuses on the effect of attractive forces on the phase behavior of lyotropic liquid crystals. The models used in the simulations described below, are all based on a hard-rod model with an appropriate attractive interactions added. We note that, in the context of thermotropic liquid crystals, many simulations have been performed using the Gay-Berne model. ${ }^{6-8}$ The latter model also takes intermolecular attraction into account-it is a generalization of the Lennard-Jones potential to nonspherical molecules and it has been shown to reproduce many liquid-crystalline phases. ${ }^{6-8}$ However, the Gay-Berne is not a good model for lyotropic liquid crystals (mainly because, in the absence of attractive interactions, it does not form a smectic phase). In fact, even for thermotropic liquid crystals, the Gay-Berne model has never successfully been used for the quantitative prediction of the phase diagram of any specific substance-in this sense, it differs both from the original Lennard-Jones model (which provides a surprisingly accurate prediction of the phase diagram of noble gases such as argon) and the hard-core models for lyotropic colloids. We therefore argue that, in the context of the present study, the hard spherocylinder model is the simplest "realistic" reference model available.

In the present study we make use of previous numerical simulations on the phase behavior of hard spherocylinders as a function of the nonsphericity $L / D .{ }^{9}$ To place the calculations discussed in this paper in a broader context, we show in Fig. 1 the complete phase diagram of hard spherocylinders in the $\left(\rho^{*}, L / D\right)$ plane. The reduced density $\rho^{*}$ is defined relative to the density at close packing, which is a function of the nonsphericity $L / D$ of the spherocylinders. In Sec. II, we investigate the phase behavior of rod-polymer mixtures using the above-mentioned hard spherocylinder model system as a reference system. The spherocylinders-polymer mixture is described by a generalized Asakura-Oosawa model which is, subsequently, treated within the framework of a thermodynamic perturbation theory previously introduced used by Lekkerkerker and Stroobants. ${ }^{10}$ In the AO model, a polymer molecule is pictured as a sphere that has a hard core interaction with the rods, but is completely penetrable to other polymer spheres. The thermodynamic perturbation theory is implemented by making use of numerical and scaled particle theory results for the hard spherocylinder reference system. The predictions for the rod-polymer phase behavior are put into perspective by comparison with the work in Ref. 10 and with some additional Gibbs-ensemble simulation of the full AO model. Subsequently, we explore an alternative approach to the problem of attractive interactions between colloidal rods: We replace the polymer-induced depletion force by an angle dependent attractive pair potential. The simulation results on this new model system for attractive rods are presented in Sec. III. Finally, in Sec. IV, the nematic-nematic phase transition is studied in more detail in the particular case of long spherocylinders with attraction, i.e., in the limit of large $L / D$ ratios. The overall phase behavior of hard rodlike particles with attractive interactions is discussed in Sec. $\mathrm{V}$. A summary of the results is, for convenience, presented in Sec. VI.

\section{MIXTURES OF SPHEROCYLINDERS AND POLYMERS}

\section{A. Depletion forces}

The addition of free nonadsorbing polymer molecules to a dispersion of colloidal particles (free and nonadsorbing as opposed to the polymers used to coat the particles for steric stabilization) may lead to phase separation into a colloid-rich and a colloid-poor phase. These polymers, when in an appropriate $(\theta-)$ solvent, behave as random coils with a radius of gyration $r_{g}$. On average, they are excluded from a shell of thickness $r_{g}$ around the colloidal particle, called the depletion zone. When two colloidal particles are brought together, these depletion zones will overlap and the total volume accessible to the polymer will increase. It is this increase in free volume that causes an effective attraction, the so-called depletion force, to induce a phase separation. The range of the attraction is directly related to the radius of gyration $r_{g}$, whereas its strength is proportional to the osmotic pressure of the polymers.

\section{B. Generalized Asakura-Oosawa model}

A simple, yet qualitatively correct model of polymercolloid mixtures was first proposed by Asakura and Oosawa $^{11}$ in the early fifties and subsequently extended by Vrij $^{12}$ and Lekkerkerker et al. ${ }^{13}$ In the Asakura-Oosawa (AO) model, the colloids are represented by hard-core par- 
ticles, while the polymers are assumed to be spheres of diameter $\sigma_{\text {pol }}=2 r_{g}$ that are mutually interpenetrable, but cannot penetrate the colloidal particles. Colloidal rods are conveniently modelled as hard spherocylinders (HSC) consisting of a cylindrical segment of length $L$ and diameter $D$ capped with two hemispheres. The colloid-colloid interaction potential $U_{\mathrm{HSC}}$ in a polymer-free system of $N$ hard spherocylinders depends on the particle positions and orientations, collectively denoted by the position vector $\mathbf{r}^{N}$ and the orientation (unit) vector $\mathbf{u}^{N}$

$$
U_{\mathrm{HSC}}\left(\left(\mathbf{r}^{N}, \mathbf{u}^{N}\right)= \begin{cases}\infty & \text { in case of rod-rod overlap } \\ 0 & \text { otherwise }\end{cases}\right.
$$

The colloid-polymer potential is $U_{c p}=\infty$ if any rodpolymer overlap occurs and $U_{c p}=0$ otherwise. As within the framework of the AO model the polymers are assumed to behave ideally, the polymer-polymer potential $U_{p p}=0$ in all circumstances.

How realistic is the Asakura-Oosawa model? The hardcore potential is a good approximation for the colloidcolloid interaction, certainly in the case of sterically stabilized particles ${ }^{3,14,15}$ but also for charged colloids dissolved in a solvent with a high ionic strength (short screening length). The assumption that the polymers are interpenetrable is only valid for dilute solutions of polymer in a $\theta$ solvent. ${ }^{16}$ The next question is, whether it is realistic to model a polymer by a sphere. Is the deformability of the (ideal) polymer not important? This question was the subject of a study performed by Meijer and Frenkel. ${ }^{17}$ They compared simulations of a hard sphere-polymer mixture using the $\mathrm{AO}$ model, with simulations using ideal, interpenetrable lattice polymers. They found that, if the radius of gyration of the polymers was less that $70 \%$ of the hard-sphere radius, the AO model works quite well.

\section{Perturbation theory}

A thermodynamic perturbation theory for the AO model of a rod-polymer mixture has been developed ${ }^{10,18}$ by considering a system of $N$ hard spherocylinders dispersed in a sea of interpenetrable polymer spheres in a volume $V$, which is kept at a fixed temperature $T$. This system is in osmotic equilibrium with a large reservoir containing the pure polymer solution, at fixed fugacity $z=\exp \left(\beta \mu_{p}\right)$, where $\mu_{p}$ is the polymer chemical potential. As we are imposing the fugacity of the system, the proper thermodynamic quantity is the grand canonical partition function

$$
\Xi\left(N, V, T, \mu_{p}\right)=\sum_{m=0}^{\infty} z^{m} Z(N, V, T, m),
$$

where $Z(N, V, T, m)$ is the canonical partition function of a system of $N$ spherocylinders and $m$ polymers. As the AO model states that the bare colloid-colloid interaction $U_{\mathrm{HSC}}\left(\mathbf{r}^{N}, \mathbf{u}^{N}\right)$ is a hard core potential that does not depend on the presence of the polymers, we can write

$$
\begin{aligned}
& Z(N, V, T, m) \\
& \quad=\frac{1}{N !} \int d \mathbf{r}^{N} d \mathbf{u}^{N} e^{-\beta U_{\mathrm{HSC}}\left(\mathbf{r}^{N}, \mathbf{u}^{N}\right)} Z_{p}\left(\mathbf{r}^{N}, \mathbf{u}^{N}, V, m\right),
\end{aligned}
$$

where $Z_{p}\left(\mathbf{r}^{N}, \mathbf{u}^{N}, V, m\right)$ is the canonical partition function of the polymers in a particular configuration of the colloidal rods. As the polymers were assumed not to interact with each other in this model, $Z_{p}\left(\mathbf{r}^{N}, \mathbf{u}^{N}, V, m\right)$ can be expressed as the $m$ th power of the one polymer partition function $Z_{p}\left(\mathbf{r}^{N}, \mathbf{u}^{N}, V, 1\right)$. Subsequent integration of $Z_{p}\left(\mathbf{r}^{N}, \mathbf{u}^{N}, V, 1\right)$ over the translational degrees of freedom of the polymer spheres yields the free volume $V_{\text {free }}\left(\mathbf{r}^{N}, \mathbf{u}^{N}\right)$, i.e., that part of the volume $V$ that is not excluded for the polymers by the hard spherocylinders

$$
Z_{p}\left(\mathbf{r}^{N}, \mathbf{u}^{N}, V, m\right)=\left[Z_{p}\left(\mathbf{r}^{N}, \mathbf{u}^{N}, V, 1\right)\right]^{m}=\left[V_{\text {free }}\left(\mathbf{r}^{N}, \mathbf{u}^{N}\right)\right]^{m}
$$

The grand canonical partition function of Eq. (2) now becomes

$$
\begin{aligned}
\Xi\left(N, V, T, \mu_{p}\right)= & \frac{1}{N !} \int d \mathbf{r}^{N} d \mathbf{u}^{N} e^{-\beta U_{\mathrm{HSC}}\left(\mathbf{r}^{N}, \mathbf{u}^{N}\right)} \\
& \times \exp \left[z V_{\text {free }}\left(\mathbf{r}^{N}, \mathbf{u}^{N}\right)\right] \\
= & \frac{1}{N !} \int d \mathbf{r}^{N} d \mathbf{u}^{N} e^{-\beta W\left(\mathbf{r}^{N}, \mathbf{u}^{N}, z\right)} .
\end{aligned}
$$

Here, $W\left(\mathbf{r}^{N}, \mathbf{u}^{N}, z\right)$ is the potential of mean force due to the presence of the polymer

$$
\begin{aligned}
\beta W\left(\mathbf{r}^{N}, \mathbf{u}^{N}, z\right) & =\beta U_{\mathrm{HSC}}\left(\mathbf{r}^{N}, \mathbf{u}^{N}\right)-z V_{\text {free }} \\
& =\beta U_{\mathrm{HSC}}\left(\mathbf{r}^{N}, \mathbf{u}^{N}\right)-\beta P_{R} V_{\text {free }}\left(\mathbf{r}^{N}, \mathbf{u}^{N}\right),
\end{aligned}
$$

where $P_{R}$ is the osmotic pressure of the polymers in the reservoir. We have used the fact that, for ideal polymers, the (osmotic) pressure and the fugacity are identical. $W\left(\mathbf{r}^{N}, \mathbf{u}^{N}, z\right)$ can be interpreted as an effective potential. Indeed, if the displacement and/or reorientation of a colloidal rod decreases the free volume available to the polymers, then $W\left(\mathbf{r}^{N}, \mathbf{u}^{N}, z\right)$ increases, i.e., becomes more repulsive. This potential is of purely entropic origin because we have assumed only hard-core interactions. Up to this point, the derivation has been exact, at least, in the context of the AO model. However, $W\left(\mathbf{r}^{N}, \mathbf{u}^{N}, z\right)$ is a many-body potential because the free volume $V_{\text {free }}$ depends not only on binary but also on multiple overlap of depletion zones. This complicates the theoretical calculation of the free volume and it is usually necessary to introduce approximations at some level.

The simplest theoretical approximation of the depletion interaction is based on thermodynamic perturbation theory. We replace $V_{\text {free }}\left(\mathbf{r}^{N}, \mathbf{u}^{N}\right)$ by its average value in the corresponding hard spherocylinder reference system. This yields the following approximation for the grand potential: 


$$
\begin{aligned}
\beta \Omega\left(N, V, T, \mu_{p}\right)= & -\ln \Xi\left(N, V, T, \mu_{p}\right) \\
\approx & -\ln \left\langle\exp \left(-\beta U_{\mathrm{HSC}}\left(\mathbf{r}^{N}, \mathbf{u}^{N}\right)\right)\right\rangle \\
& -z\left\langle V_{\mathrm{free}}\left(\mathbf{r}^{N}, \mathbf{u}^{N}\right)\right\rangle \\
= & \beta F_{\mathrm{HSC}}-z_{p} \alpha V .
\end{aligned}
$$

Here, the brackets stand for an ensemble average over the colloid configurations, $F_{\text {HSC }}$ is the free energy of the hard spherocylinders and $\alpha$ is the average fraction of the total volume available for the polymers. Both $F_{\text {HSC }}$ and $\alpha$ depend on the density $\rho=N / V$ and on the orientational and/or (partial) translational order in the system. Indeed, $F_{\text {HSC }}$ and $\alpha$ have different values in, for example, an isotropic and a nematic phase at the same density $\rho$.

From the grand potential one can calculate the osmotic pressure $P$ and the chemical potential $\mu_{s c}$ of the colloidal spherocylinders

$$
\begin{aligned}
& \beta P=-\beta\left(\frac{\partial \Omega}{\partial V}\right)_{N, \mu_{p}, T}=\beta P_{\mathrm{HSC}}+z\left(\alpha-\rho \frac{\partial \alpha}{\partial \rho}\right), \\
& \beta \mu_{s c}=\beta\left(\frac{\partial \Omega}{\partial N}\right)_{V, \mu_{p}, T}=\beta \mu_{\mathrm{HSC}}-z \frac{\partial \alpha}{\partial \rho} .
\end{aligned}
$$

Together with expressions for the Helmholtz free energy $F_{\text {HSC }}$ and for the available free volume fraction $\alpha$ these equations can be used for a theoretical study of the phase behavior of a hard spherocylinder-polymer mixture.

\section{Calculations}

Lekkerkerker and Stroobants ${ }^{10}$ used the formalism outlined in Sec. II C to calculate the phase behavior of rodpolymer mixtures. Scaled particle theory (SPT) provided the equation of state of the hard spherocylinder reference system and of the free volume fraction $\alpha$ required in the perturbation theory and this in both the isotropic and nematic phase. The phase behavior was investigated systematically as a function of the aspect ratio $L / D$ of the HSC's and of the parameter $q=\sigma_{p} / D$ which determines the actual range of the depletion interaction.

For HSC's with $L / D=5$, the case we will focus on in the present study, demixing was found to occur in the isotropic phase for large $q$ values, i.e., for long polymers. This corresponds roughly with the results of the experiments on sterically stabilized boehmite rods. ${ }^{4}$ The critical point of the I-I binodal shifts to higher fugacities upon lowering $q$ and becomes metastable with respect to the I-N transition for $q$ $<0.65$. For sufficiently short polymers so that $q<0.1$, a demixing transition was found in the nematic phase.

However, as was already noted in Ref. 10, the theoretical prediction of such a $\mathrm{N}-\mathrm{N}$ transition for $L / D=5$ should be treated with some caution. The $\mathrm{N}-\mathrm{N}$ transition occurs at such high volume fraction that neglecting alternative transitions involving more highly ordered (liquid-)crystalline phases does not seem justified. SPT does indeed not take into account the smectic and the solid phase, that are found in the pure $L / D=5$ HSC system. ${ }^{9,19,20}$ In the present study we
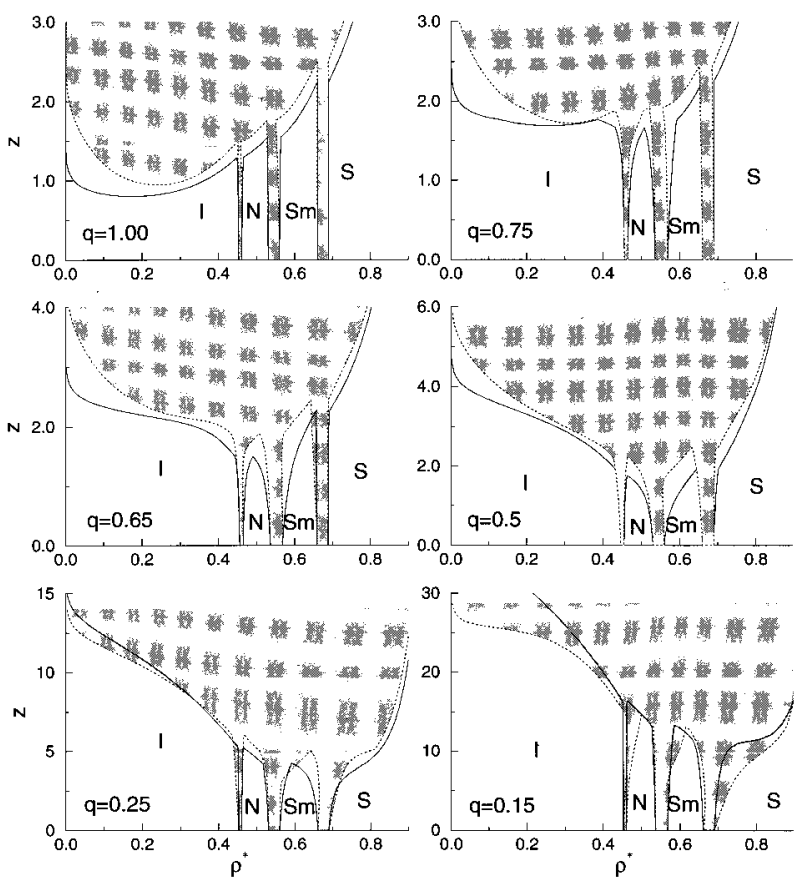

FIG. 2. Phase diagrams for a system of spherocylinders with $L / D=5$ mixed with polymers of different diameter ratio $q$, as predicted by thermodynamic perturbation theory. The reference system is the hard spherocylinder model, and the perturbation was evaluated by numerical simulation of the reference system (dotted lines, gray area) or by the SPT expression Eq. (11) (solid lines).

make an attempt to predict the complete phase behavior of HSC's with $L / D=5$ in the presence of polymer, by combining thermodynamic perturbation theory and simulation results.

The thermodynamic perturbation theory (Sec. II C) requires as input the equation of state of the hard spherocylinder reference system and of the free volume fraction $\alpha$. Reference 9 contains the necessary information about the equation of state of HSC's. In addition, we measure the free volume fraction $\alpha(\rho)$ as a function of the density $\rho$ using the Widom insertion technique. ${ }^{21}$ In the Widom method, it is attempted to insert polymers (represented by hard spheres) at random positions in the simulation box. The fraction of insertions that does not result in an overlap corresponds to the free volume fraction. In practice, we measure the distance $r$ to the nearest surface of a spherocylinder. A polymer with a radius larger than $r$ will overlap with the spherocylinder, while a smaller polymer will fit. We made a histogram of the insertion probability, and hence the free volume fraction $\alpha$, as a function of $\rho$ and $q$. We fitted this free volume data to a polynomial in $\rho$ and $q$ and used Eqs. (9) and (10) in combination with the simulation results for $L / D=5^{9}$ to calculate the two-phase coexistence curves. The resulting phase diagrams in the $\left(\rho^{*}, z\right)$ plane are presented in Fig. 2 for $q$ $=0.15,0.25,0.50,0.65,0.75$, and 1.0.

The advantage of the perturbation approach is that there is no need to simulate the polymers explicitly: It is sufficient to just measure the free volume available for a polymer in a pure hard spherocylinder system. However, despite a direct 
simulation of the full AO model is bypassed, a whole new set of simulations is still required to measure $\alpha(\rho)$. It is therefore very tempting to combine, within the framework of the perturbation theory, a simple theoretical expression for $\alpha(\rho)$ and the numerical equation-of-state date of Ref. 9. SPT actually provides such an expression ${ }^{22,23}$ and the free volume fraction $\alpha$ in an isotropic HSC fluid as a function of the volume fraction $\phi$ is now given by

$$
\begin{aligned}
\alpha= & (1-\phi) \exp \left[-A\left(\frac{\phi}{1-\phi}\right)-B\left(\frac{\phi}{1-\phi}\right)^{2}\right. \\
& \left.-C\left(\frac{\phi}{1-\phi}\right)^{3}\right],
\end{aligned}
$$

where

$$
\begin{aligned}
& A=\frac{6 \gamma}{3 \gamma-1} q+\frac{3(\gamma+1)}{3 \gamma-1} q^{2}+\frac{2}{3 \gamma-1} q^{3}, \\
& B=\frac{1}{2}\left(\frac{6 \gamma}{3 \gamma-1}\right)^{2} q^{2}+\left(\frac{6 \gamma}{3 \gamma-1}+\frac{6(\gamma-1)^{2}}{(3 \gamma-1)^{2}}\right) q^{3}, \\
& C=\frac{2}{3 \gamma-1}\left(\frac{12 \gamma(2 \gamma-1)}{(3 \gamma-1)^{2}}+\frac{12 \gamma(\gamma-1)^{2}}{(3 \gamma-1)^{2}}\right) q^{3}, \\
& \gamma=L / D+1 .
\end{aligned}
$$

Furthermore, we will use this SPT result for the isotropic phase, in the nematic, smectic, and solid phases of the HSC system as well. Although this might seem rather drastic, it should be stressed that a similar approach has previously lead to satisfactory results in the case of the hard spherepolymer mixtures. ${ }^{13}$ The phase diagrams, obtained by using a "hybrid", numerical/theoretical input for the perturbation theory, are shown in Fig. 2 for comparison with the results for the "exact"' numerical input.

Finally, we should comment on the slight difference in philosophy underlying the present implementation of the perturbation theory and the SPT approach of Ref. 10. In the latter case, the equilibrium (isotropic, nematic) phase at a particular density $\rho$ is found by minimizing the grand potential Eq. (8) with respect to the one-particle orientational distribution function $f(\mathbf{u})$. In contrast, in the present study, the equilibrium (isotropic, nematic, smectic, solid) phases are obtained by simulating the polymer-free HSC reference system. Formally, this comes down to establishing the equilibrium phase in the rod-polymer mixture at some density $\rho$ through minimization of the Helmholtz free energy $F_{\mathrm{HSC}}$ instead of the full grand potential $\Omega$. This implies, physically, that any structural changes are neglected that might be induced in those equilibrium phases by the polymer molecules.

\section{E. Results}

Figure 2 shows the phase diagrams in the $\left(\rho^{*}, z\right)$ plane for HSC's with $L / D=5$ and different polymers such that $q$ $=0.15,0.25,0.50,0.65,0.75$, and 1.0., using the "exact", numerical input for the perturbation theory. These figures show the thermodynamically stable regions of the isotropic, nematic, smectic A and solid phase denoted by I, N, SmA and $\mathrm{S}$, respectively. The phases are separated from each other by first-order coexistence regions indicated by a grey area. Two coexisting phases will have equal $z$, because $z$ is the fugacity of the polymer reservoir that is in equilibrium with both phases.

The phase boundaries obtained with a "hybrid", numerical/theoretical input for the perturbation theory are displayed in the same Fig. 2. The predictions of the scaled particle theory are in good qualitative agreement with the numerical approach. This is remarkable, considering the approximate nature of the SPT treatment of the free volume fraction $\alpha$.

In Fig. 2(a) the phase diagram for $q=1.00$ is depicted. The isotropic fluid phase contains the familiar I-I binodal curve ending in a critical point. At a fugacity higher than $z_{\text {crit }}=0.95$ a phase separation occurs between a vapor phase with a low density of spherocylinders, and a high density liquid phase. Upon increasing the fugacity of the polymers, the liquid becomes metastable with respect to the nematic phase and we find gas-nematic coexistence. At still higher fugacity the nematic phase also disappears and there is a two phase gas-smectic coexistence region. Eventually, at $z$ $=2.5$ the gas-smectic is preempted by the crystallization transition. We find that the densities of the coexisting phases at the $\mathrm{I}-\mathrm{N}, \mathrm{N}-\mathrm{SmA}$, and the $\mathrm{SmA}-\mathrm{S}$ transitions, hardly change with the polymer fugacity.

In Fig. 2(b) we consider the situation for a smaller polymer radius of $q=0.75$. As expected, the shorter interaction range results in a shift of the critical point of the I-I demixing transition to higher densities. Moreover, the transition occurs at higher polymer fugacity. We note that the I-N coexistence density gap widens (slightly) at higher polymer fugacity. The density region where the nematic phase is stable does, therefore, decrease at these high polymer fugacity. The I-I-N triple point occurs at somewhat higher fugacity. The I-N-SmA and $\mathrm{I}-\mathrm{SmA}-\mathrm{S}$ triple points are hardly affected.

When we decrease the range of interaction to $q=0.65$, the critical point of the I-I demixing curve disappears. The I-I binodal has moved to higher fugacity where the phase transition is preempted by the (successively) I-N, I-SmA, and $\mathrm{I}-\mathrm{S}$ transitions. The $\mathrm{I}-\mathrm{N}-\mathrm{SmA}$ and $\mathrm{I}-\mathrm{SmA}-\mathrm{S}$ triple points still occur at roughly the same polymer fugacity, although close to the triple points, all three first order phase coexistence regions widen somewhat.

For $q=0.50$ the widening of the $\mathrm{N}-\mathrm{SmA}$ transition becomes very pronounced for $z>1.5$. The I-N-SmA triple point increases in fugacity faster than the $\mathrm{N}-\mathrm{SmA}-\mathrm{S}$ triple point. This shift in the relative location of triple points continues and for $q=0.25$ the $\mathrm{N}-\mathrm{SmA}-\mathrm{S}$ occurs at lower $z$ than the $\mathrm{I}-\mathrm{N}-\mathrm{S}$ triple point. The interaction range is now small enough to affect the $\mathrm{SmA}-\mathrm{S}$ transition. The shape of the coexistence curve in the solid phase is typical of a system approaching an isostructural solid-solid transition. However, the interaction ranges is still too large to induce such a phase separation inside the solid phase. As discussed in Ref. 24, in the case of spherical colloids, this transition occurs only if the interaction range $\delta$ becomes less than $7 \%$ of the hard- 


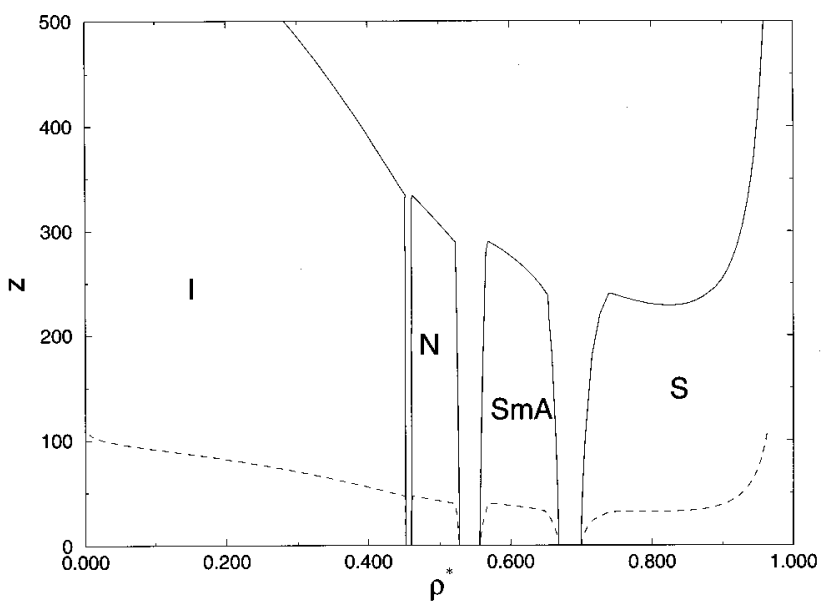

FIG. 3. Phase diagrams for a system of spherocylinders with $L / D=5$ mixed with polymers of diameter ratio $q=0.05$ (solid line) and $q=0.10$ (dashed line), as predicted by thermodynamic perturbation theory. The reference system is the hard spherocylinder model, and the perturbation was evaluated by the SPT expression Eq. (11) (solid lines). The $q=0.10$ phase diagram does not show a solid-solid phase separation, whereas the $q=0.05$ phase diagram clearly has a critical point in the solid phase.

sphere diameter. The last plot of Fig. 2 shows the phase diagram for $q=0.15$. The SmA-S transition has become even wider, and the $\mathrm{I}-\mathrm{N}-\mathrm{S}$ and the $\mathrm{N}-\mathrm{SmA}-\mathrm{S}$ triple points both move to higher $z$ values, but their relative position is hardly affected. Although, because of numerical inaccuracy in the fitting of the free volume function $\alpha$, the "exact", numerical perturbation theory did not result in reasonable phase diagrams for smaller values of $q$, we were able to compute the phase diagrams for $q=0.05$ and $q=0.10$ with the SPT-"hybrid" method. This phase diagram is plotted separately in Fig. 3 and shows clearly the existence of a solid-solid transition for $q=0.05$. As the $q=0.10$ phase diagram does not have a critical point, the solid-solid transition becomes stable somewhere in between $q=0.05$ and $q$ $=0.10$ which is in agreement with the $7 \%$ result of Ref. 24 .

Summarizing, the following global picture emerges from the perturbation theory calculations. For large polymers we have an isotropic fluid-fluid phase separation ending in a critical point which shifts to larger $z$ and $\rho^{*}$ and is preempted when the radius becomes smaller then $q=0.6$. The I-N-SmA and I-SmA-S triple points occur at $z=2.2$ and 2.5 , respectively, and are not very sensitive to the polymer size for $q>0.6$. For lower $q$ both triple points move to higher $z$, but the $\mathrm{I}-\mathrm{N}-\mathrm{SmA}$ overtakes the $\mathrm{I}-\mathrm{SmA}-\mathrm{S}$ triple point. For $q<0.45$ the situation changes and we have and $\mathrm{I}-\mathrm{N}-\mathrm{S}$ and a N-SmA-S triple point. For the low $q$ values the I-N, N-SmA, and SmA-S coexistence regions fan out for high $z$. We find no evidence for an $\mathrm{N}-\mathrm{N}$ transition, as predicted by the SPT approach of Ref. 10. In particular, for $q=0.1$ the scaled particle theory predicts a $\mathrm{N}-\mathrm{N}$ critical point at $\rho^{*}=0.6$. However, the present simulations suggest that, at that density, the nematic phase is no longer stable with respect to the smectic. The perturbation theory that we use becomes less accurate at high $z$, because it neglects any structural changes induced by the polymer. Still, this limita- tion is unlikely to affect our conclusion that a polymerinduced $\mathrm{N}-\mathrm{N}$ phase separation will not occur for rods with a nonsphericity $L / D=5$. After all, the density range over which the nematic phase is stable, is quite limited: There is hardly space for an $\mathrm{N}-\mathrm{N}$ binodal. We therefore expect a nematic-nematic phase separation to take place for larger elongation of the spherocylinder, where the nematic phase extends over a much wider density range.

For the same reason, it seems equally unlikely that a smectic to smectic transition will occur for $L / D=5$. Moreover, increasing the aspect ratio of the rods will do little to facilitate the occurrence of a smectic-smectic transition, as the density range where the smectic phase is stable hardly changes with $L / D$ (see Fig. 1). It is tempting to speculate that polydispersity of the rods may change these conclusions: polydispersity in the length of the rods enhances the nematic stability, thus making a $\mathrm{N}-\mathrm{N}$ separation more likely. In contrast, polydispersity in the diameter of the spherocylinders widens the smectic range: ${ }^{25}$ This may favor the occurrence of a demixing transition in the smectic phase.

It is very encouraging to find that the results obtained by using a "hybrid" numerical/theoretical input for the perturbation theory are in reasonable agreement with those obtained with an "exact" numerical input. This strongly suggests that it must be possible to gain physical insight in the overall phase behavior of rod-polymer mixtures, i.e., for arbitrary $L / D$ and $q$ values, by combining the numerical equation of state data of Ref. 9 and the SPT expression for the free volume fraction $\alpha$. We note, however, that the phase diagram computed using perturbation theory is quite sensitive to small statistical errors in the free energy and equation of state of the reference system.

\section{F. Full AO model simulation of I-I coexistence}

In the above we used perturbation theory to estimate the phase behavior of the Asakura-Oosawa model for rodpolymer mixtures. The crucial assumption in the perturbation theory is that we can use the properties of the hard spherocylinder reference fluid to compute the free-volume fraction $\alpha$ at finite fugacity. To check if this assumption is justified, we have to perform simulations of the full Asakura-Oosawa model at finite $z$. Although this is certainly feasible for individual state points, such calculations would become prohibitively expensive if we wish to compute phase diagrams for different values of $q$ and different aspect ratios of the rods. The reason is that, in a full (off-lattice) simulation of the AO model, every polymer has to be simulated separately. The number of particles needed for such simulation is quite large, certainly for the small polymers at high fugacities. As an example we can estimate the number of polymers in a nematic phase of spherocylinders of $L / D=5$ at a density $\rho^{*}$ $=0.5$ in equilibrium with a reservoir with polymers of diameter $\sigma_{p}=0.15$. As can be seen in Fig. 2 , the polymer fugacity can easily be equal to $z_{p}=10$. The free volume fraction for this density and $q$ value is approximately 0.4 . This corresponds to an equilibrium polymer density in the nematic phase of $\rho_{p}=z \phi=4$. As the volume of the simulation box 
has to be larger than $(2(L+D))^{3}$ to avoid multiple overlaps of the spherocylinders, the minimum number of polymers needed in such a simulation is $N_{p}=4 \times 12^{3} \approx 7 \times 10^{3}$. This is a lower bound, as the polymers tend to drive the spherocylinders together and create more space for themselves. Moreover, due to the large particle concentration, the acceptance probability of moving a spherocylinder in a sea of polymers is dropping dramatically.

For $L / D=5$ spherocylinder systems with larger polymers $\left(\rho_{p} \approx 1\right)$ the number of polymer particles which is necessary to induce an isotropic fluid-fluid phase separation is of the order of only a few thousand. We therefore can attempt to conduct Gibbs-ensemble simulations of the AO model in the isotropic phase at finite $z$. The Gibbs-ensemble technique enables one to obtain the phase equilibria directly, instead of via a more expensive thermodynamic integration. ${ }^{26-28}$ Of course, the technique is limited to low density phases in order to keep the acceptance ratio of spherocylinder exchanges reasonable. However, because the location of I-I separation binodal is very sensitive to approximations, the direct simulation of this phase equilibrium provides a good test for the validity of the perturbation theory.

Even in the isotropic phase the acceptance ratio of exchanging a spherocylinder is still extremely low, because it will nearly always cause overlap with an polymer. To improve this acceptance ratio we make use of a Monte Carlo configurational bias technique developed for a similar problem. ${ }^{29}$

We performed Gibbs-ensemble simulations for the $L / D$ $=5$ system with polymers of diameter $q=1.0$ and $q=0.8$ at different polymer fugacities. The overall reduced density of spherocylinders was $\rho^{*}=0.21$ and $\rho^{*}=0.30$ for the $q=1.0$ and $q=0.8$ system, respectively. The total number of spherocylinders in the system was 360 . We did not fix the number of polymers in the system, but rather kept their fugacity $z$ constant. During the simulations the number of polymers varied from 3000 to 6000 . As a test, we measured the chemical potential of the polymers and the spherocylinders using Widoms particle insertion technique ${ }^{21}$ during a simulation. The chemical potential $\mu_{p}$ of the polymers is directly related to the fugacity by $z=\exp \left(\beta \mu_{p}\right)$. We checked that the actual chemical potentials of the two species remained constant in both phases.

For equilibration of every system $100000 \mathrm{MC}$ cycles were executed, whereas 100000 cycles were performed for data collection. During a cycle every particle on average was moved once. In addition the average cycle contained 300 attempted particle exchanges and 1 volume exchange between the two phases. The maximum step sizes of the particle moves were adjusted in order to keep the acceptance probability around $30 \%-40 \%$. The acceptance probability of HSC exchanges which involved the configurational bias Monte Carlo step was $\pm 0.05 \%$. The acceptance probability for the insertion of polymers was $\pm 70 \%$ in the low density phase and $\pm 15 \%$ in the high density phase.

The results of the Gibbs-ensemble simulations are plotted in Fig. 4. For comparison, both the numerical and the
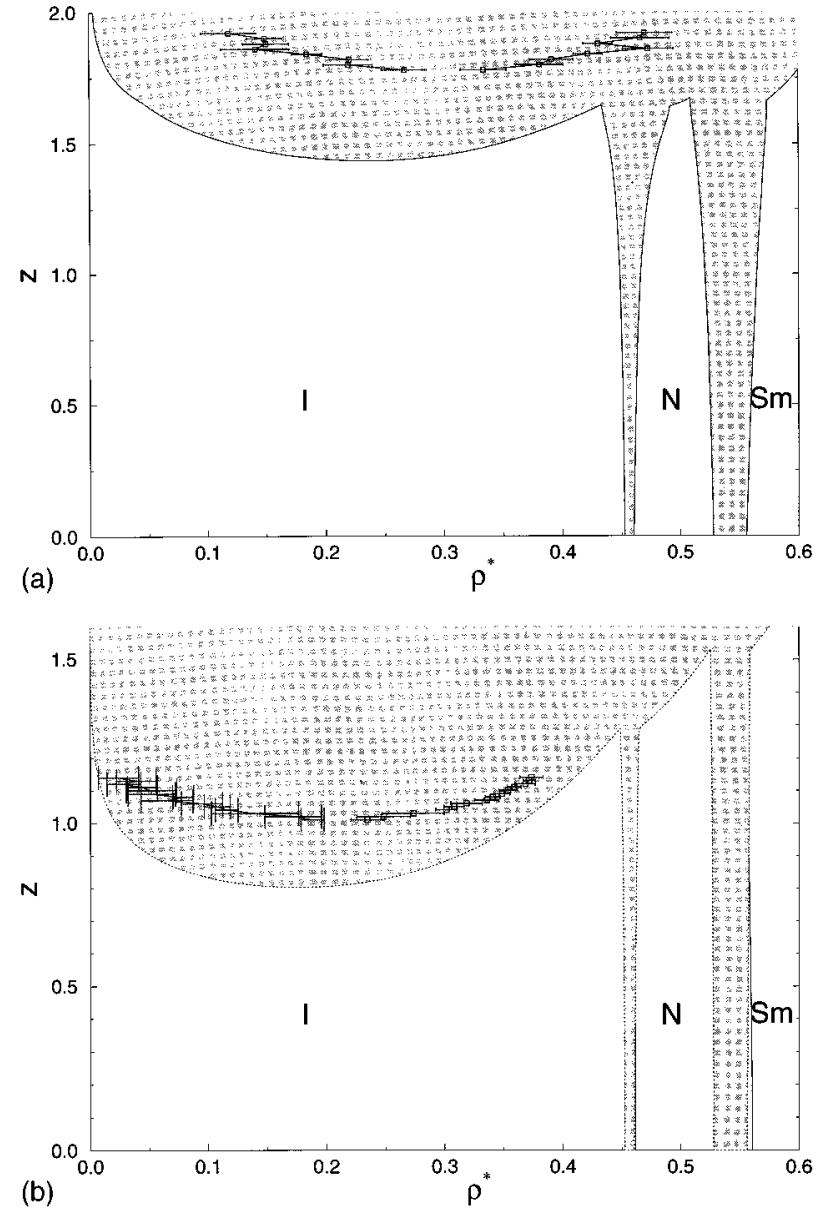

FIG. 4. Isotropic fluid-fluid coexistence curve for a system of spherocylinders with $L / D=5$ mixed with polymers of diameter ratio $q=0.8$ (a) and $q=1.0$ (b), obtained by modified Gibbs-ensemble simulations. For comparison, the phase diagrams as predicted by thermodynamic perturbation theory (see Fig. 2) are included.

SPT-"hybrid" perturbation theoretical phase diagrams are included as well. The figure clearly shows that the approximation of the perturbation theories is rather good. The Gibbs-ensemble simulations yield a much flatter binodal than is predicted by the mean-field theory. Such behavior is well known, although it should not be taken to imply that Gibbsensemble simulations on finite systems correctly reproduce the 3D-Ising critical behavior of the infinite system. The critical density of the Gibbs-ensemble results seems to be in between the SPT-"hybrid" and the numerical one. Nevertheless, on basis of the good agreement between the theoretical results and the Gibbs-ensemble simulations, we can conclude that the use of the hard spherocylinder fluid as a reference system in thermodynamic perturbation theory yields a reasonable approximation for the phase behavior of mixtures of spherocylinders and polymers within the AO model.

\section{SPHEROCYLINDERS WITH PAIRWISE-ADDITIVE ATTRACTION}

\section{A. The angle dependent pair potential}

In the previous section we showed that for reasonably large polymers it is possible to check the assumptions under- 
lying the perturbation theory by performing a direct Gibbsensemble simulation of the isotropic fluid-fluid coexistence of the AO model ensemble simulation at finite fugacity $z$. However, in the case of smaller polymers the critical point of the I-I binodal shifts to higher density and becomes metastable with respect to the I-N transition. Because of the high densities involved it becomes almost impossible to perform direct Gibbs ensemble simulation of the I-N or I-SmA coexistence. Furthermore, as we mentioned in the previous section the number of polymer particles needed to reach the necessary high fugacity becomes of the order of $10^{4}$. Calculations involving such large numbers of particles would become prohibitively expensive if we wish to compute phase diagrams for different values of $q$ and different aspect ratios of the spherocylinders.

To avoid simulating the full polymer-colloid model, while retaining the effect of the depletion interaction on the structure of the colloidal suspension, we constructed a model of hard spherocylinders with a pairwise additive attractive "depletion" potential that approximates the real potential of mean force $W$. The most important feature of this potential is that it must be dependent on relative orientation of the rods. The depletion attraction is strong $(\sim q L)$ when the spherocylinders are parallel, but much weaker $\left(\sim q^{2}\right)$ for perpendicular rods.

The most direct way to compute the depletion pair potential is to calculate the overlap volume between the depletion zones of the two spherocylinders as a function of distance and relative orientation, and multiply this volume by the polymer osmotic pressure. This procedure yields an effective pair potential that can be used in simulations of the phase diagram. In the case of spherical colloids mixed with small polymers, this approach predicts a phase diagram that is in reasonable agreement with the "exact" phase diagram. ${ }^{17,30}$ Although the depletion pair potential for spherocylinders could be computed in the same way, the resulting function would not be cheap to compute during a simulation. As the computation of the pair potential is the most time-consuming part of the program, it is useful to devise an effective pair potential that resembles the depletion interaction, but is computationally cheap. To this end, we assume that the spherocylinder can be considered as a continuous distribution of (overlapping) spheres of diameter $D$, with their centers distributed uniformly on a line segment of length $L$. The individual spheres interact through a square-well potential. The total pair potential of the two spherocylinders is then a sum (or actually, an integral) of all the individual contributions, and is expressed as

$$
\begin{aligned}
V(r)= & -\epsilon \int_{-L / 2}^{L / 2} \int_{-L / 2}^{L / 2} d \lambda_{i} d \lambda_{j} H((\delta+D) \\
& \left.-\left|\Delta \mathbf{r}_{i j}+\lambda_{i} \mathbf{u}_{i}-\lambda_{j} \mathbf{u}_{j}\right|\right) .
\end{aligned}
$$

Here, $\Delta \mathbf{r}_{i j}$ denotes the relative position of the centers of mass of the two hard spherocylinders $i$ and $j$, with orientations specified by the unit vectors $\mathbf{u}_{i}$ and $\mathbf{u}_{j}$, respectively. The range of depletion interaction $(\delta)$ is a measure for the polymer diameter $\sigma_{p}$. The well depth $\epsilon$ can be interpreted as
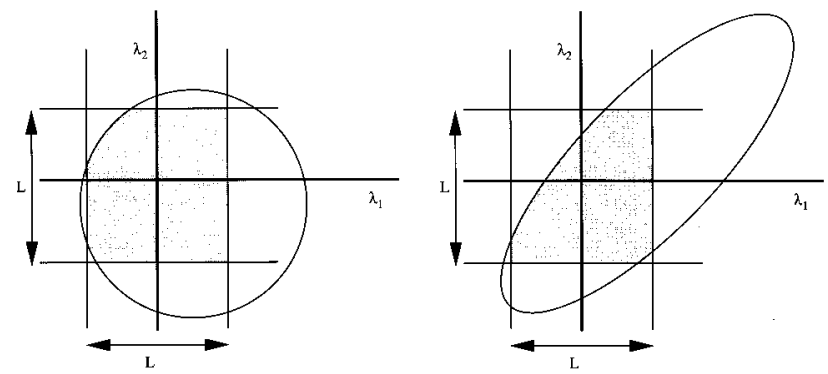

FIG. 5. Geometrical representation of the computation of the effective depletion interaction between two spherocylinders [Eq. (13)]. On the left, the situation for two perpendicular spherocylinders is depicted. The $\lambda_{1}$ and $\lambda_{2}$ axes denote the distance from the center of mass along a cylinder axis. The circle indicates the $\left(\lambda_{1}, \lambda_{2}\right)$ points for which the distance equals $\delta$ $+D$. Inside this circle the distance is smaller than $\delta+D$. As the integral in Eq. (13) is bounded by the length of the spherocylinders, the total interaction is proportional to the cross section of the $L \times L$ square and the ellipse. On the right, the angle between the spherocylinder is less than 90 degrees. The circle therefore changes into an ellipse, with its major axes along the lines $\lambda_{1}=\lambda_{2}$ and $\lambda_{1}=-\lambda_{2}$. The interaction is still proportional to the area bounded by the ellipse and the condition $\left|\lambda_{1}\right|<L / 2$ and $\left|\lambda_{2}\right|<L / 2$.

a measure for the polymer concentration. The Heaviside step-function $H(x)$ is defined in the usual manner

$$
H(x)=\left\{\begin{array}{ll}
1 & x \geqslant 0 \\
0 & x<0
\end{array} .\right.
$$

The evaluation of the integral in Eq. (13) can be reduced to a simple geometrical problem. First, consider the interaction between two infinitely long cylinders. If the cylinders are mutually perpendicular, the integral will reduce to the calculation of the surface of a circle with radius $\sqrt{(\delta+D)^{2}-\Delta^{2}}$, where $\Delta$ is the minimum distance between the lines that run through the cylinder axes. As the angle between the cylinders becomes smaller, this circle changes into an ellipse by scaling one of the ellipse axes by $1 / \sqrt{1-\mathbf{u}_{i} \mathbf{u}_{j}}$. The integral diverges for parallel, infinite cylinders, because every point along the cylinder gives a finite contribution. However, the integral in Eq. (13) is bounded by the finite length of the spherocylinders. This is translated in the geometrical picture as cuts through the ellipse, as shown in Fig. 5. If we multiply the area enclosed by the ellipse and/or the cuts of the $L / 2$ bounds by the well depth $\epsilon$ we obtain the total interaction potential.

A quantity of particular interest is the maximum interaction between two spherocylinders. The interaction between two particles is largest when they are oriented parallel with their centers of mass at the minimum distance $(D)$

$$
\begin{aligned}
V_{\max }(D) & =-\epsilon\left(2 L \sqrt{(\delta+D)^{2}-D^{2}}-\left((\delta+D)^{2}-D^{2}\right)\right) \\
& =-\epsilon\left(2 L \sqrt{\delta^{2}+2 \delta D}-\left(\delta^{2}+2 \delta D\right)\right)
\end{aligned}
$$

In Fig. 6 we show the behavior of this pair potential for a few relative orientations and distances of the two spherocylinders. 

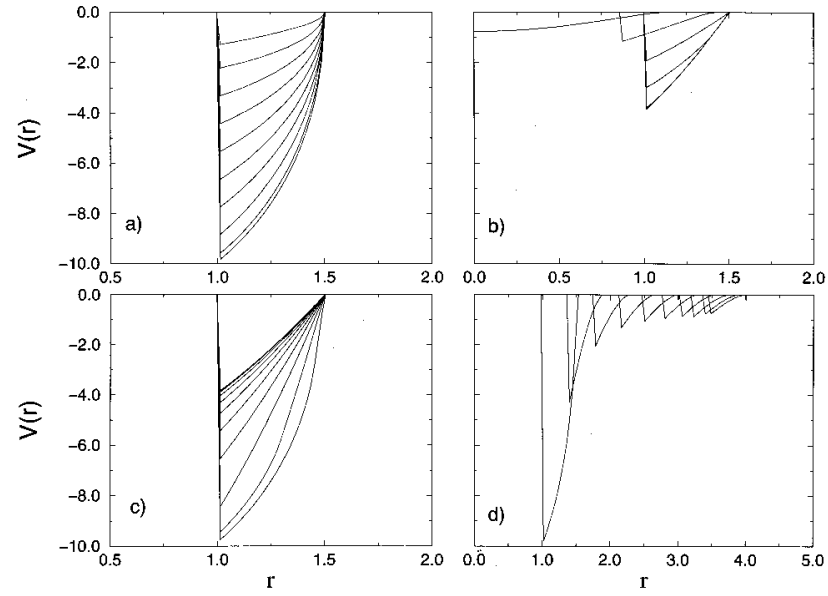

FIG. 6. Generalized square-well interaction potentials $V(r)$, as a function of distance $r$ for various orientations and center-of-mass distances of two spherocylinders with $L / D=5$. One spherocylinder is fixed at the origin oriented along the $z$ axis, while the position and orientation of the second is varied. (a) Parallel spherocylinders, with the center of mass of the second spherocylinder shifted along the $z$ direction. From the bottom to the top the curves denote potentials for a shift of $z / D=0,0.5,1.0,1.5,2.0,2.5,3.0,3.5$, 4.0, 4.5, and 5.0. (b) Perpendicular spherocylinders, with the center of mass of the second spherocylinder shifted along the $z$ direction. From the bottom to the top the curves denote potentials for a shift of $z / D=0,0.5,1.0,1.5$, 2.0, 2.5, 3.0, 3.5, 4.0, 4.5, and 5.0. (c) Two spherocylinders with zero $z$ shift, the second one rotated around the line through the centers of mass over an angle $\theta$. From the bottom to the top the curves denote potentials for an angle of $\theta=0,10,20,30,40,50,60,70,80$, and 90 degrees. (d) Two spherocylinders with zero $z$ shift, the second one rotated over an angle $\theta$ in the plane through the line connecting the centers of mass and the $z$ axis. From the bottom to the top the curves denote potentials for an angle of $\theta=0,10,20$, $30,40,50,60,70,80$, and 90 degrees.

\section{B. Simulation}

We performed NVT Monte Carlo simulation on a system of 480 spherocylinders with $L / D=5$ interacting through the generalized square-well potential described in the previous section, for $\delta=0.1,0.3,0.5$, and 1.0. The density ranged from $\rho^{*}=0.1$ in the isotropic phase to $\rho^{*}=0.66$ in the smectic phase. The reduced well depth $\epsilon^{*}=\epsilon / k T$ is a measure for the polymer concentration. In our simulations, it ranged from $0-2$. Configurations of the system at high densities were generated by slowly expanding a perfectly aligned, close packed smectic structure. Care was taken to avoid that the system became stuck in glassy configurations, a problem that was particularly severe at low temperatures (large $\epsilon^{*}$ ). We measured the potential energy as a function of $\rho^{*}$ and $\epsilon^{*}$ and fitted it to a polynomial of the form

$$
E\left(\rho^{*}, \epsilon^{*}\right)=\sum_{i, j}^{3,2} \rho^{* i} \epsilon^{* j} .
$$

The free energy of the system could be obtained via thermodynamic integration

$$
\begin{aligned}
F\left(\rho^{*}, \epsilon^{*}\right) & =F_{\mathrm{HSC}}\left(\rho^{*}\right)+\int d \epsilon^{*}\left(\frac{\partial F}{\partial \epsilon^{*}}\right) \\
& =F_{\mathrm{HSC}}\left(\rho^{*}\right)+\int d \epsilon^{*} \frac{\langle E\rangle_{\epsilon^{*}}}{\epsilon^{*}},
\end{aligned}
$$

where $\langle E\rangle$ is the average potential energy measured during the Monte Carlo simulations and $F_{\mathrm{HSC}}$ denotes the free energy of the hard spherocylinder reference system. This free energy can be obtained by integration along the equation of state in the different phases, where the reference free energies were taken from the results of Ref. 9.

At large $\epsilon^{*}$ (low $T$ ), the Helmholtz free energy $F\left(\rho^{*}, \epsilon^{*}\right)$ may exhibit an inflection point, signalling the occurrence of a first-order phase transition within a single phase (e.g., isotropic-isotropic). Alternatively, the increased attraction may shift the phase coexistence boundaries of the I-N, N-SmA, or SmA-S transitions. In either case, we can estimate the density of the coexisting phases by using a double tangent construction.

\section{Results}

The results of the thermodynamic integration are displayed in Fig. 7 for different values of $q=\delta / D$. The graphs are plotted in the $\rho^{*}, \epsilon^{*}$ plane instead of the usual $\rho^{*}, T$ plane because the well depth $\epsilon^{*}$ is more directly related to the fugacity of the polymers in the system. In this way one can compare the phase diagrams with those of Sec. II C. We note, however, that the pair potential that we use is not completely equivalent to the Asakura-Oosawa interaction. For this reason we focus on the qualitative features of the phase diagram. For $q=1$ the range of the potential is equal to the diameter of the spherocylinder and we see a phase separation between two isotropic phases ending in a critical point. The density region where the nematic phase is stable becomes narrower as $\epsilon$ increases and it ends in an isotropic-nematicsmectic triple point at a fugacity of $\epsilon^{*} \approx 0.23$. This is lower than the location of the $\mathrm{I}_{1}-\mathrm{I}_{2}-\mathrm{N}$ triple point at $\epsilon^{*} \approx 0.34$. In contrast, the results of the perturbation theory in Sec. II C indicate that for $q=1$ the $\mathrm{I}-\mathrm{N}-\mathrm{SmA}$ triple point has a higher fugacity than the $\mathrm{I}_{1}-\mathrm{I}_{2}-\mathrm{N}$ one. Apparently, the attractive pair potential destabilizes the nematic phase with respect to the smectic phase.

At higher fugacity the smectic phase is expected to become metastable with respect to the solid. Although we have not included the solid in our simulation, it is likely that the general picture is similar to that found in Sec. II C.

The perturbation theory of Sec. II C predicts that, for smaller values of $q$, the isotropic-isotropic transition will shift to higher fugacity and density and will, eventually be preempted by the isotropic-nematic transition. Indeed, for $q=0.5$ we find that the I-I transition has already disappeared. The I-N and $\mathrm{N}-\mathrm{SmA}$ transitions widen at higher $\epsilon^{*}$. The I-N-SmA triple point is located at $\epsilon^{*}=0.3$. For still smaller $q$, the picture remains much the same. The I-N and N-SmA transitions widen at high $\epsilon^{*}$, the triple point moves to higher $\epsilon^{*}$ and lower $\rho^{*}$. This last feature was not predicted by the perturbation theory.

We do not observe a nematic-nematic or smecticsmectic phase separation. In Sec. II C, we argued that a nematic-nematic or smectic-smectic transition will, most likely, be preempted by a transition to a phase with a different symmetry. However, for large $L / D$, the range of stability 

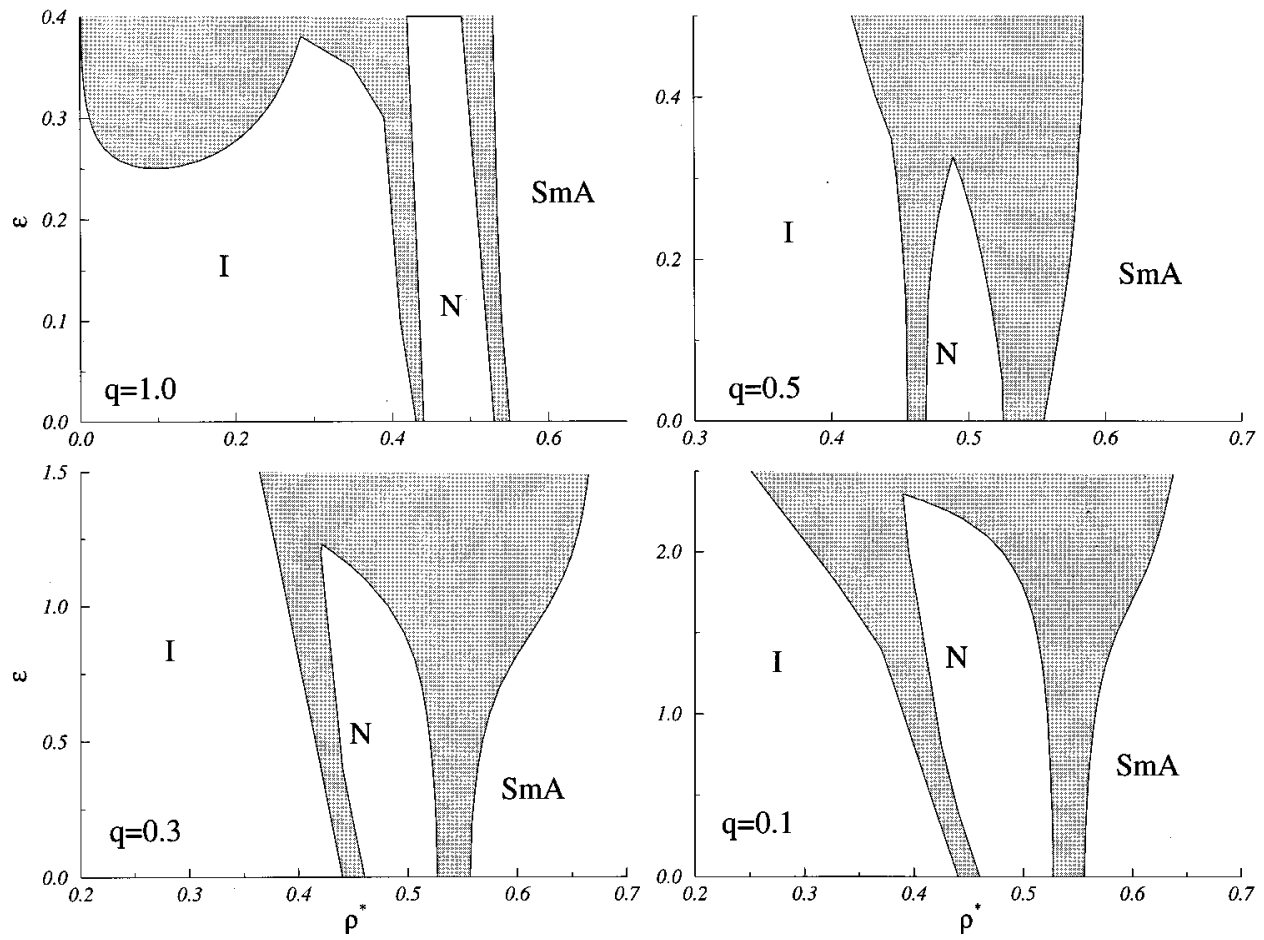

FIG. 7. Simulated phase diagrams for $L / D=5$ spherocylinders with an attractive generalized square-well potential [Eq. (13)]. The diagrams are plotted in the $\rho^{*}, \epsilon^{*}$ plane to facilitate comparison with the Asakura-Oosawa model of rod-polymer mixtures.

of the nematic phase will become very large. Under those circumstances, it is likely that a $\mathrm{N}-\mathrm{N}$ phase transition becomes possible

\section{LONG SPHEROCYLINDERS WITH ATTRACTION}

\section{A. Nematic-nematic coexistence in the Onsager limit}

The existence of a nematic-nematic phase transition in systems of rods with an attractive potential was already predicted in the early work of Flory ${ }^{31}$ and later by Khokhlov and Semenov. ${ }^{32}$ They estimated that a nematic-nematic transition can occur for rods with $L / D>15$, but will be metastable for smaller elongations.

To investigate the possibility of a polymer-induced N-N transition for suspensions of long rods, we start from the known phase behavior of a system of infinitely long ("'Onsager") rods. ${ }^{9}$ To include the depletion interaction, we must extend the generalized square-well interaction discussed in Sec. III to long rods in the limit $L / D \rightarrow \infty$. We consider the situation that the system is in the dense nematic phase, or in an even more highly ordered phase, where the rods are almost perfectly aligned. This allows us to use an affine transformation to map the long spherocylinder system onto skewed right cylinders with $L / D=1$. Note that the same affine transformation will map an Asakura-Oosawa sphere onto an infinitely thin disk. All the depletion interactions are therefore limited to points on the same $x y$ plane. The total depletion potential can be obtained by integrating all in- plane interactions along the two rods. (i.e., by integrating $z$ in the interval where both cylinders are intersected by the same $x y$ planes).

$$
V(r)=-\epsilon \int_{-1 / 2}^{1 / 2} d z H((\delta+D)-|\Delta r(z)|),
$$

where $\Delta r(z)$ is the distance between the axes of the two rods in the $x y$ plane at height $z$. Of course, we should take the effect of the finite length of the rods into account. As in Sec. III A, this integral can be given a simple geometrical interpretation.

In Fig. 8 we consider two particles $i$ and $j$ at a center of mass distance $r_{i j}$. The top surface of skewed cylinder $i(j)$ is shifted with respect to the bottom by an amount $\mathbf{u}_{i}\left(\mathbf{u}_{j}\right)$. We can deform the coordinate frame such that the axis of one particle (say $i$ ) is along the $z$ axis. Projecting cylinder $i$ along the $z$ axis, we can draw a circle of radius $\delta+D$ around the particles axis, which shows the range of the square well potential. In the same figure, we project that fraction of the axis of particle $j$ that is located between the top and bottom planes of particle $i$. We denote the length of this segment by $\alpha$. Next, we project this line segment on a plane perpendicular to the $z$ axis. The resulting line segment may intersect the circle that delimits the range of the square well potential. Let us denote by $\beta$, the fraction of the projected line segment that lies within this circle. The interaction strength of particle $i$ and $j$ is then given by $\epsilon \alpha \beta$. Obviously, the maximum interaction strength, $V(D)=-\epsilon$, is found for two parallel particles at contact and at equal height. 


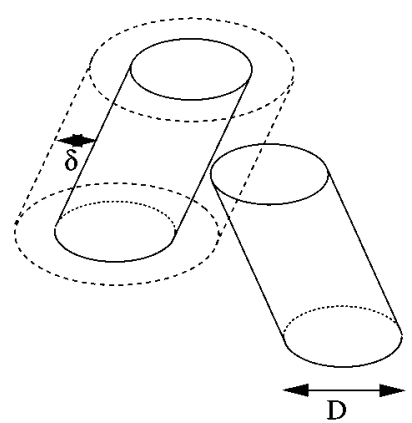

a)

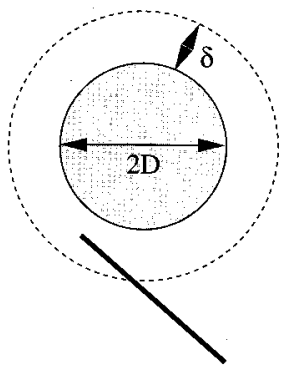

b)
FIG. 8. Geometrical representation of the computation of the polymer induced depletion potential for spherocylinders in the limit $L / D \rightarrow \infty$. On the left, two interacting scaled spherocylinders are depicted. The range of the square well is indicated by the dashed spherocylinder. The fraction of the length of the right cylinder that is inside this dashed volume, is proportional for the interaction potential, according to Eq. (19). (b) is a projection along the cylinder axis of one of the scaled spherocylinders. The dashed circle gives the boundary of the square well interaction. The thick line segment is the projection of that part of the axis of the other cylinder that is located between the top and bottom surfaces of the first cylinder. We denote the (unprojected) length of this segment by $\alpha$. The fraction of the line segment inside the dashed circle circle is denoted by $\beta$. The value of the integral in Eq. (19) is given by $\alpha \times \beta$.

We performed simulations of the Onsager system for the nematic and smectic phases with densities between $\rho^{*}$ $=0.1$ and $\rho^{*}=0.7$. The width of the potential well was chosen to be $\delta=1$, while the well depth $\epsilon^{*}$ was varied between $\epsilon^{*}=0$ to $\epsilon^{*}=2.5$. We measured the average potential energy as a function of $\rho^{*}$ and $\epsilon^{*}$ and constructed a freeenergy surface for the nematic and smectic phases by thermodynamic integration, starting from the known equation of state of the pure Onsager particles. A nematic-nematic transition shows up as nonconvex behavior of the volume dependence of this free energy. The nematic-smectic transition, although presumably continuous in the pure Onsager system, becomes first order upon introducing an attraction. The calculated phase diagram is shown in Fig. 9. Clearly, the

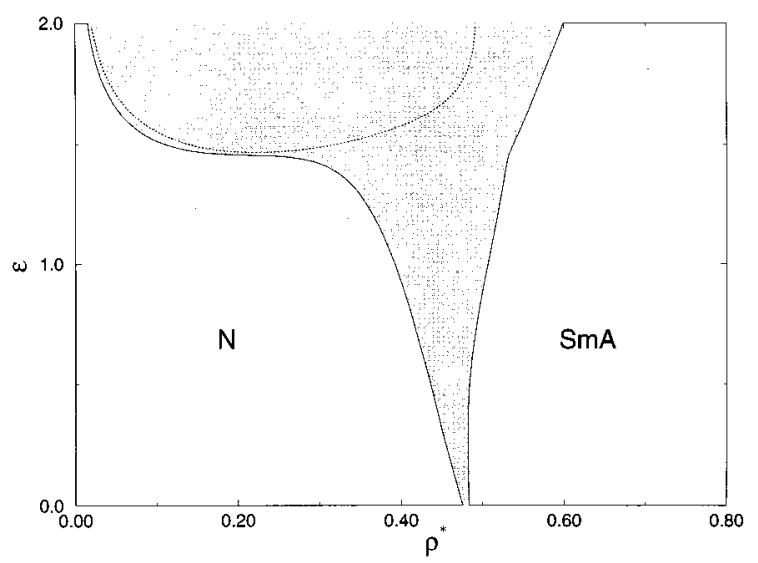

FIG. 9. The phase diagram for a system of infinitely long hard rods, with an attractive interaction given by Eq. (19). The range of the attraction is $q$ $\approx \delta=1$. The diagram is plotted in the $\rho^{*}, \epsilon^{*}$ plane to make comparison with the rod-polymer mixture possible. The dashed curve is the metastable $\mathrm{N}-\mathrm{N}$ binodal.

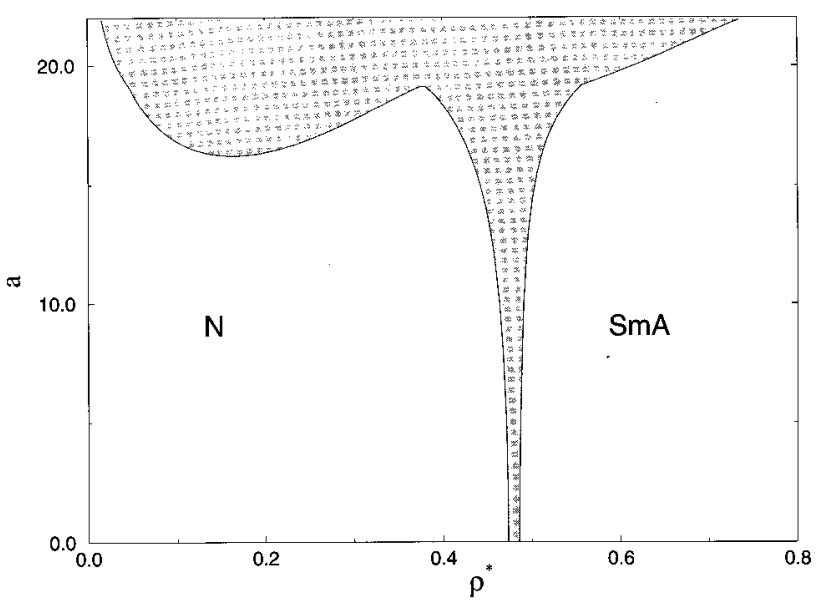

FIG. 10. Phase diagram for a system of infinitely long spherocylinders with an infinitely long ranged, infinitely weak, attractive potential. Note that the nematic-nematic binodal has become stable with respect to the $\mathrm{N}-\mathrm{SmA}$ transition, be it over a narrow range in "temperature" (as measured by the strength of the attraction, $a$ ).

nematic-nematic separation is (just) metastable with respect to the nematic-smectic transition. We expect that the $\mathrm{N}-\mathrm{N}$ binodal shifts to lower reduced density and $\epsilon^{*}$ as the range of attraction becomes larger. For sufficiently long ranged attractions, there could be a stable $\mathrm{N}-\mathrm{N}$ binodal. To check this, we studied a system with infinitely long-ranged (but infinitely weak) attraction. That is, we analyze the "van der Waals" limit of the Onsager system plus attraction.

\section{B. Infinitely long-ranged attraction for long rods}

Thermodynamic perturbation theory becomes exact in the limit of infinitely weak, infinitely long attraction. ${ }^{33}$ This is the basis of the success of the van der Waals model. We can use a similar mean-field theory to examine the phase behavior of spherocylinders with an infinitely long-ranged interaction in the limit $L / D \rightarrow \infty$. The van der Waals free energy is the sum of the free energy of the hard core reference system and the change in free energy due to the attractive interactions. In the limit of infinitely long-ranged attractions, mean field theory is exact, and the perturbation is simply proportional to the density

$$
F_{\mathrm{vdW}}=F_{\mathrm{HSC}}-a \rho^{*} .
$$

Here, the parameter $a$ is a measure for the strength of the attractive interactions or, equivalently, a measure for the (inverse) temperature. In the polymer-colloid case, $a$ is a measure for the polymer fugacity. The phase diagram for the Onsager system with long-ranged attraction is displayed in Fig. 10. Clearly there now is a nematic-nematic transition, which ends in a critical point at a density of $\rho^{*} \approx 0.19$. We also include the $\mathrm{I}-\mathrm{N}$ and the $\mathrm{N}-\mathrm{SmA}$ transition in the figure, in order to show that the $\mathrm{N}-\mathrm{N}$ is stable with respect to these transitions. As before, the $\mathrm{N}-\mathrm{Sma}$ transition, widens at large values of $a$ (higher fugacity). In the Onsager model, the I-N transition is located at $\rho^{*}=0$ and is therefore not visible in the figure. In summary, it is indeed possible to have a stable 


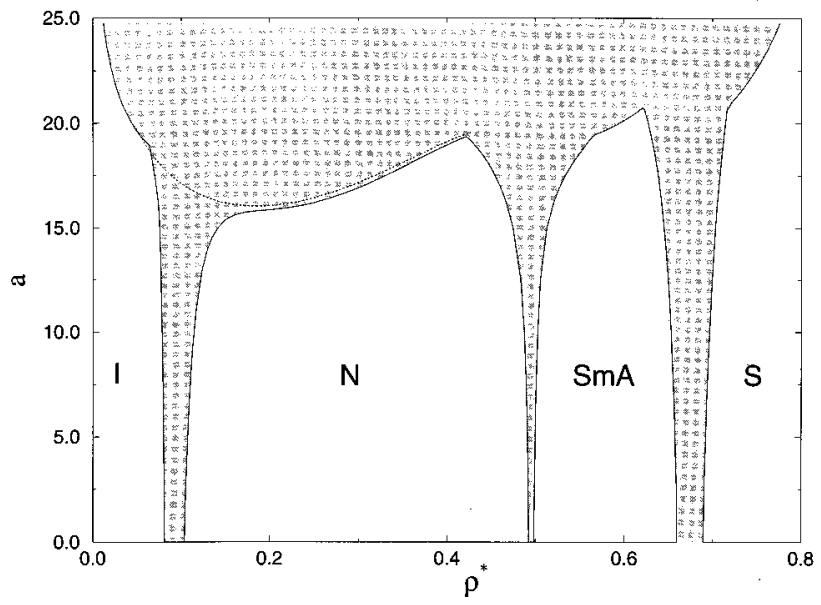

FIG. 11. Phase diagram for a $L / D=40$ spherocylinder system with an infinitely long ranged, infinitely weak, attractive potential. The nematicnematic binodal is preempted by the isotropic-nematic transition.

nematic-nematic phase separation in a system of infinitely long hard spherocylinders with attraction, provided that the interaction is sufficiently long ranged.

If we now consider the van der Waals limit for spherocylinders with a large but finite aspect ratio, the situation changes slightly. From the work of Ref. 9 we know that the isotropic-nematic transition moves to higher densities as we move away from the Onsager limit, while the density of the $\mathrm{N}-\mathrm{SmA}$ transition is hardly affected. This opens the possibility that the isotropic-nematic transition interferes with the $\mathrm{N}-\mathrm{N}$ transition. And this is precisely what happens: Figure 11 shows that the $\mathrm{N}-\mathrm{N}$ phase separation in the limit of infinitely long-ranged attractions, is preempted by the I-N transition. The figure suggests that the nonsphericity $L / D=40$ is close to the limit where $\mathrm{N}-\mathrm{N}$ transitions are possible. Only slightly longer rods would be needed, to recover the $\mathrm{N}-\mathrm{N}$ binodal.

The perturbation theory calculations indicate that shortening the range of attraction (i.e., decreasing the value of $q$ ) will move the critical demixing point to higher densities and will also lead to a reappearance of the $\mathrm{N}-\mathrm{N}$ transition, until, in its turn, it is pre-empted by the $\mathrm{N}-\mathrm{SmA}$ transition at small $q$ values.

This picture corresponds qualitatively to the prediction of Khokhlov and Semenov ${ }^{32}$ that the N-N transition is only possible for rods longer $L / D=15$. The significant difference with our lower limit $L / D=40$ cannot be explained by the isotropic attraction, because both theory and simulation use the same isotropic (exact) mean field potential. Therefore, the discrepancy must be due to the way the orientation dependent interactions are treated. In the theory they are approximated by a simple anisotropic potential, whereas in the simulations are determined by the (many body) excluded volume of the rods.

Let us next consider depletion interactions under conditions that correspond to the van der Waals description for pairwise additive forces. In this limit, depletion forces are simple too, but yield results that are very different from the ones obtained with pairwise additive van der Waals interactions. To see this, consider a system of very large AO spheres and hard rods. In the limit that the radius of the AO spheres goes to infinity, this system will always demix completely, because the excess chemical potential of the spheres, i.e., the reversible work needed to introduce an $\mathrm{AO}$ sphere of volume $V_{s}$ in a system of hard spherocylinders at pressure $P$, is to leading order simply equal to $P V_{s}$. As $V_{s}$ diverges, so will the excess chemical potential. Hence, the solubility of spheres in spherocylinders (and, as is easy to verify, the solubility of spherocylinders in spheres), drops to zero at all finite pressures. As is explained in Ref. 29, the condition for coexistence is now simply that the pressure of the AO phase and the hard spherocylinder system are equal. The pressure of the AO phase is given by the ideal gas law. We can therefore consider the fluid of AO spheres as an "osmotic piston" acting on the spherocylinder system. As we increase the density of the $\mathrm{AO}$ spheres, the pure spherocylinders are compressed and will undergo the complete sequence of phase transitions from isotropic all the way to the crystalline solid. But, as the AO phase is always isotropic, coexistence between phases of the same symmetry is limited to the isotropic phase. In the limit that the AO spheres are infinitely large compared to the spherocylinders, the I-I critical point will be located at the point $P=0, \rho=0$. However, for finitesize spheres, this critical point will be observed at finite pressure and at low, but finite, concentration of the spherocylinders.

This behavior should be contrasted with that of spherocylinders with van der Waals attraction, discussed above. This difference in behavior should have noticeable experimental consequences. Consider two systems of rodlike colloids with the same aspect ratio. In one system the attraction is due to dispersion forces (i.e., van der Waals-like) and in the other, we assume that attraction is caused by depletion interaction. We consider the case that the range of attraction is at least comparable to the diameter of the rods. We can prepare the two systems at the same effective "temperature" by choosing the conditions such that they have the same second virial coefficients (expressed in units of the corresponding hard-core system). Under those conditions, the two systems will not obey a law of corresponding states and, in particular, the phase diagrams are likely to differ considerably.

\section{DISCUSSION}

On basis of the available results, we can give a rough sketch of the expected regions where demixing transitions should occur in the $q, L / D$ plane. In Fig. 12 we have combined our present knowledge about the stability regions of the phase separations in the different phases as a function of the polymer interaction range $q$ and the colloid nonsphericity $L / D$. The curves give a rough indication of the regions in the $q, L / D$ plane where the depletion interaction induces a binodal in any one of the phases. The I-I critical point starts to appear for hard spheres around $q=0.3$. For rods with a nonsphericity $L / D=5$, the minimum interaction range needed to 


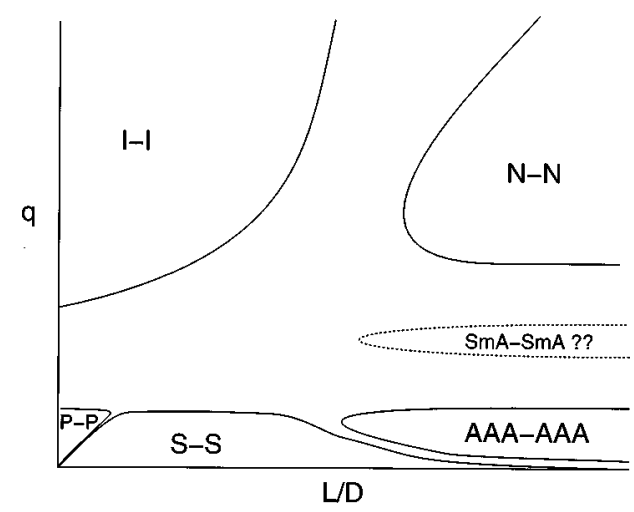

FIG. 12. Schematic summary of the regions of in the phase diagram of spherocylinder polymer mixtures, where demixing into two phases with the same symmetry can occur. See the text.

induce an I-I phase transition, has increased to $q=0.6$ and it probably increases steeply with increasing aspect ratio. In fact, beyond a certain nonsphericity (less than $L / D=40$ ), stable I-I transition is not even possible in the limit $q \rightarrow \infty$. The nematic-nematic transition first appears for systems with an aspect ratio that is large enough to support a nematic phase over a reasonable density range. For finite $L / D$ values, the region where a $\mathrm{N}-\mathrm{N}$ transition is possible is bounded on both the low and high $q$ side. The high- $q$ boundary rises steeply with increasing $L / D$, and for large $L / D$, the region where an $\mathrm{N}-\mathrm{N}$ transition can occur is only bounded for low $q$. In our simulations, we have not observed a SmA-SmA phase separation, but there is no a priori reason to exclude it either. However, as the range of stability of the smectic phase is never very large, we expect the SmA-SmA transition, if it occurs at all, be confined to a narrow "window" in the $q, L / D$ plane. A possible location for this window has been sketched in Fig. 12. For very short-ranged attractions (low values of $q$ ), we expect to observe an isostructural solid-solid phase transition separation over the entire $L / D$ range. This solid-solid transition was discussed in Ref. 24 for the case of hard spheres. For spherocylinders, we expect to see a solid-solid transition in the plastic phase for small $L / D$ (say, $L / D \leqslant 0.3$ ). For larger $L / D$ values it is possible to have a solid-solid transition between two ordered solid phases ( $A B C$ stacking). Finally, for large $L / D$ values $(L / D$ $\geqslant 10)$ the solid-solid phase separation takes place between two $A A A$ structures. For hard spheres, the $S-S$ transition in two and three dimensions appears at approximately the same range of the attractive interaction. If we take the point of view that a solid of long spherocylinders resembles a twodimensional crystal, then it seems reasonable to surmise that the range of $q$ values for which the solid-solid transition is possible, depends only little on $L / D$. It should, however, be stressed that the analogy between a solid of almost very long, well aligned spherocylinders in the $A A A$ phase, and a twodimensional crystal of disks, is not perfect, because the orientational entropy of the rods can never be neglected. There are gaps between the three different regions of solid-solid transitions. In those gaps, the effect of the depletion interaction is to widen the density gap between two coexisting solid phases of different symmetry. Finally, it is tempting to speculate that the effect of the depletion interaction on the phase behavior of the $A A A$-stacked solid, may be even more complex. In a two-dimensional system, the solid-solid critical point induces a hexatic pocket in the phase diagram. ${ }^{34}$ Possibly, the solid-solid transition in the $A A A$-stacked phase favors the formation of a liquid-crystalline hexatic- $B$ phase.

\section{SUMMARY}

We performed simulations to study the phase diagram of hard, rodlike particles with attractive interactions. We distinguish the case that the attractions are due to pairwise additive forces or to the (nonadditive) depletion force that is caused by the addition of nonadsorbing polymers. We first consider this system in the context of the generalized AsakuraOosawa model. Lekkerkerker and Stroobants have used a combination of thermodynamic perturbation theory and scaled particle theory to predict the phase diagram of this model system. We first tested the quality of the scaledparticle against numerical data, and found the theory to be surprisingly good, even under conditions where it might be expected to fail. In addition we compare the thermodynamic perturbation theory itself with direct (Gibbs ensemble) simulations of the isotropic-isotropic phase coexistence. And, again, we find that the theory gets the qualitative features of the phase diagram right.

Subsequently, we studied a system of spherocylinders with nonsphericity $L / D=5$ using an effective pairwise additive attraction to model the depletion interaction. The shape of the pair potential was chosen such that, in the limit of short-ranged attraction, it reproduces the effect of polymer depletion. The range of this generalized square-well potential $(q)$ is a measure for the size (radius of gyration) of the polymer. For large polymer sizes, an isotropic-isotropic phase separation takes place that ends in a critical point. This critical point shifts to higher density and higher polymer fugacity as the polymer radius is reduced. Eventually (for $q<0.6$ ), the I-I transition is preempted by the I-N transition. The perturbation theory results and our simulations on spherocylinders with a generalized square-well interaction indicate that, for $L / D=5$ there is no phase separation in the nematic or smectic phase. We expect that, for very small $q(\leqslant 0.05)$, there is a solid-solid transition at high densities, but we have not verified this. For large $q$, the I-N-SmA triple point occurs at a lower fugacity than the I-Sma-S point, but for $q<0.45$, the I-SmA-S triple point disappears and we are left with a $\mathrm{I}-\mathrm{N}-\mathrm{SmA}$ and a $\mathrm{N}-\mathrm{SmA}-\mathrm{S}$ triple point. The latter is located at the lower fugacity.

A nematic-nematic phase separation is possible for large $L / D$. In the limit $L / D \rightarrow \infty$, the $\mathrm{N}-\mathrm{N}$ transition is not yet possible for $q=1$. We estimate that $\mathrm{N}-\mathrm{N}$ coexistence becomes possible for $q$ values larger than one. For finite $L / D$ values, the $q$ window of stability is also bounded from the upper side, where the I-N transition will eventually preempt the $\mathrm{N}-\mathrm{N}$ phase separation.

If a smectic-smectic separation occurs at all, it is likely to be confined to a narrow window in the $q, L / D$ plane. At 
high densities and small values of $q$, it is possible to have isostructural solid-solid transitions, involving the plastic solid, $A B C$-stacked solid, and $A A A$-stacked solid, for small, intermediate and large $L / D$ values, respectively.

We expect that polydispersity of the spherocylinders will have a pronounced effect on the phase behavior. Polydispersity in the length will stabilize the nematic phase and make a nematic-nematic transition possible at shorter (average) lengths. In contrast, polydispersity in the diameter of the spherocylinders will stabilize the smectic A phase, and could thereby favor the occurrence of a smectic-smectic phase transition.

\section{ACKNOWLEDGMENTS}

The work of the FOM Institute is part of the research program of FOM and is made possible by financial support from the Netherlands Organization for Scientific Research (NWO).

${ }^{1}$ S. S. Cohen, J. Biol. Chem. 144, 353 (1942).

${ }^{2}$ S. Fraden, in Observation, Prediction and Simulation of Phase Transitions in Complex Fluids, NATO ASI Series C, Vol. 460, edited by M. Baus, L. F. Rull, and J. P. Ryckaert (Kluwer Academic, Dordrecht, 1995).

${ }^{3}$ P. A. Buining, C. Pathmamamnoharan, J. B. H. Jansen, and H. N. W. Lekkerkerker, J. Am. Ceram. Soc. 74, 1013, (1991).

${ }^{4}$ J. Buitenhuis, L. N. Donselaar, P. A. Buining, A. Stroobants, and H. N. W. Lekkerkerker, J. Coll. Interface Sci. 175, 46 (1995).

${ }^{5}$ P. A. Buining, Y. S. J. Veldhuizen, C. Pathmamamnoharan, and H. N. W. Lekkerkerker, Colloid Surfaces 64, 47 (1992).

${ }^{6}$ D. J. Adams, G. R. Luckhurst, and R. W. Phippen, Mol. Phys. 61, 1575 (1987).

${ }^{7}$ E. De Miguel, L. F. Rull, M. K. Chalam, and K. E. Gubbins, Mol. Phys. 74, 405 (1991).

${ }^{8}$ E. De Miguel, E. Martin del Rio, J. T. Brown, and M. P. Allen, J. Chem. Phys. 105, 4234 (1996).

${ }^{9}$ P. G. Bolhuis and D. Frenkel, J. Chem. Phys. 107, 666 (1997).
${ }^{10}$ H. N. W. Lekkerkerker and A. Stroobants, Nuovo Cimento D 16, 949 (1994).

${ }^{11}$ S. Asakura and F. Oosawa, J. Chem. Phys. 22, 1255 (1954).

${ }^{12}$ A. Vrij, Pure Appl. Chem. 48, 471 (1976).

${ }^{13}$ H. N. W. Lekkerkerker, W. C.-K. Poon, P. N. Pusey, A. Stroobants, and P. B. Warren, Europhys. Lett. 20, 559 (1992).

${ }^{14}$ A. K. van Helden, J. W. Jansen, and A. Vrij, J. Colloid Interface Sci. 81, 354 (1981).

${ }^{15}$ P. N. Pusey, in Liquids, Freezing and the Glass Transition, Les Houches, session LI, edited by D. Levesque, J. P. Hansen, and J. Zinn-Justin (NorthHolland, Amsterdam, 1991).

${ }^{16}$ P. J. FLory, Principles of Polymer Chemistry (Cornell University Press, Ithaca, 1953).

${ }^{17}$ E. J. Meijer and D. Frenkel, J. Chem. Phys. 100, 6873 (1994).

${ }^{18}$ H. N. W. Lekkerkerker, P. Buining, J. Buitenhuis, G. J. Vroege, and A. Stroobants, in Observation, Prediction and Simulation of Phase Transitions in Complex Fluids, NATO ASI Series C, Vol. 460, edited by M. Baus, L. F. Rull, and J. P. Ryckaert (Kluwer Academic, Dordrecht, 1995).

${ }^{19}$ D. Frenkel, H. N. W. Lekkerkerker, and A. Stroobants, Nature (London) 332, 882 (1988).

${ }^{20}$ S. C. McGrother, D. C. Williamson, and G. Jackson, J. Chem. Phys. 104, 6755 (1996).

${ }^{21}$ B. Widom, J. Chem. Phys. 39, 2808 (1963).

${ }^{22}$ M. A. Cotter, Phys. Rev. A 10, 625 (1974).

${ }^{23}$ M. A. Cotter, J. Chem. Phys. 66, 1098 (1977).

${ }^{24}$ P. G. Bolhuis and D. Frenkel, Phys. Rev. E 50, 4880 (1994).

${ }^{25}$ R. van Roij, Ph.D. thesis, Universiteit Utrecht, The Netherlands, 1996.

${ }^{26}$ A. Z. Panagiotopolous, Mol. Phys. 61, 813 (1987).

${ }^{27}$ M. Stapleton, A. Z. Panagiotopolous, and N. Quirke, Mol. Phys. 63, 527 (1988).

${ }^{28} \mathrm{Ph}$. de Smedt, B. Smit, and D. Frenkel, Mol. Phys. 68, 931 (1989).

${ }^{29}$ P. G. Bolhuis and D. Frenkel, J. Chem. Phys. 101, 9869 (1994).

${ }^{30}$ A. P. Gast, C. K. Hall, and W. B. Russel, J. Colloid Interface Sci. 96, 251 (1983).

${ }^{31}$ P. J. Flory, Proc. R. Soc. London 234, 73, (1956).

${ }^{32}$ A. R. Khokhlov and A. N. Semenov, J. Stat. Phys. 38, 161, (1985).

${ }^{33}$ P. C. Hemmer and J. L. Lebowitz, in Critical Phenomena and Phase Transitions, edited by C. Domb and M. Green (Academic, New York, 1976).

${ }^{34}$ P. Bladon and D. Frenkel, Phys. Rev. Lett. 74, 2519 (1995). 